\title{
Hybrid Filters based Denoising of Medical Images using Adaptive Wavelet Thresholding Algorithm
}

\author{
Shruti bhargava \\ Research scholar ,DKNMU
}

\author{
Ajay Somkuwar \\ professor,MANIT Bhopal
}

\begin{abstract}
several new techniques are developed within the previous couple of years that convalesce results on spacial filters by take away the noise additional with success whereas protective the sides within the information. a trifle of those techniques used the background from partial differential equations and process fluid dynamics like level set strategies, non-linear isotropous and anisotropic diffusion. A little range of techniques pooled impulse removal filters with native adaptive filtering within the rework domain to require out not solely white and mixed noise, however additionally their mixtures. so as to diminish the noise gift in medical pictures several techniques area unit procurable like digital filters (FIR or IIR), adaptive filtering strategies etc. nonetheless, digital filters and adaptive strategies are often applied to signals whose applied math characteristics area unit stationary in several cases. currently the moving ridge rework has been incontestable to be great tool for non-stationary signal analysis. we have a tendency to take PSNR and MSE as a potency issue to envision the effectiveness of planned denoising formula.
\end{abstract}

\section{Keywords}

PSNR (Peak Signal to Noise Ratio), MSE (Mean Square Error), DWT (Discrete Wavelet Transform), Wavelet De-noising, Normal Thresholding, Adaptive Thresholding, Soft and Hard Thresholding.

\section{INTRODUCTION}

Medical info, serene of clinical knowledge, pictures and different physiological signals, has become Associate in Nursing necessary a part of a patient's care, throughout screening, within the diagnostic stage and within the dealing part. Over the past 3 decades, flying developments in info technology (IT) \& Medical Instrumentation has expedited the enlargement of digital medical imaging. This growth has in the main involved X-raying (CT), resonance Imaging (MRI), the various digital imaging processes for vascular, vas and distinction imaging, diagnostic technique, diagnostic ultrasound imaging, nuclear medical imaging with Single gauge boson Emission X-raying (SPECT) and antielectron Emission imaging (PET). All these progression ar manufacturing ever-increasing quantities of pictures. These pictures ar altered from typical photographic pictures primarily as a result of they tell internal anatomy as divergent to a picture of surfaces. These Medical pictures have be in possession of distinctive set of challenges. though our focus during this paper are going to be on twodimensional pictures, three-dimensional (volume) pictures, time-varying two-dimensional pictures (movies), and timevarying three-dimensional pictures ar ofttimes used clinically as imaging modalities are getting additional refined.The noise idiom could also be additive, increasing or combination of each. just in case of medical pictures wel've got along additive and increasing noise relying upon the modalities worn for image acquisition. during this analysis, we are going to take pictures contaminated with mathematician noise, Laplacian noise and Poisson noise.

\section{RELATED WORK}

In the field of medical image denoising ample methodology square measure developed a number of ways we tend to mentioned here. recently developed linear transformation freelance part analysis (ICA) methodology with well-known linear transformation ways like principal part analysis(PCA), correlational analysis, and projection pursuit in their paper titled "Survey on freelance part Analysis" [21]. Their paper surveyed distinction functions and algorithms for ICA. As elements within the ICA illustration square measure l'as freelance as possiblel' additionally as l'as non-Gaussian as possiblel', this methodology is essentially most well-liked to cut back the selection between estimating all the freelance elements at an equivalent time, and estimating solely a set of them, presumably one-by-one.another author given a unique approach to image denoising victimisation accommodative principal elements in paper, "Adaptive Principal elements and Image Denoising" [19]. Authors given this new technique assumptive that the image is corrupted by additive white mathematician noise. Their formula performs well in terms of image visual fidelity and PSNR values, against some denoising algorithms like Hidden Markoff Tree Models, the spatially accommodative image denoising formula, the SI-Adaptive Shrink. wave thresholding technique is extensively used for denoising medical pictures. this concept and changed these coefficients to get rid of noise from the information. Authors evaluated many denoising ways in their paper titled, "Medical Image Denoising victimisation wave Thresholding" [20]. Paper showed that the NormalShrink methodology provides higher PSNR as compared to the opposite wavelet-based techniques VisuShrink, BayesShrink Authors additionally demonstrate that garrote shrinkage offers blessings over each arduous and soft shrinkage. Performance Comparison of Median and Wiener Filter in Image De-noising" [22]. They compared performance of Median and Wiener Filters in Image de-noising for various kinds of noise like mathematician noise, Salt \& Pepper noise and Speckle noise. And simulation results show that Wiener Filter performs higher for Speckle and mathematician clattering image. Whereas Median filter performs higher for Salt \& Pepper clattering image.

\section{PROPOSED ALGORITHM}

Firstly, i would like to debate a trifle regarding dataset that we tend to area unit planning to use for analysis motivation, we tend to in the main target medical resonance imaging knowledge for our denoising situation, we are going to conjointly take a look at our sculpt to totally different alternative medical pictures conjointly like CT, PET Ultrasound pictures.

In order to prevail over the unwell influence of noise and shading, there's a requirement to require them into contemplation once choosing the brink being employed. On the opposite hand, this can be associate degree undoable mission in world context, since nobody threshold will work the whole image. This ends up in the conclusion, that a supplementary native threshold should be used. The section property will apportion a number of cautious assumptions, and in line with them manufacture an acceptable threshold for the pixels within 
the atmosphere. the final designation of a threshold is written within the following manner: $T=T[x, y, p(x, y), f(x, y)]$

Where $f(x, y)$ is the gray level of point $(x, y)$ in the original image, and $\mathrm{p}(\mathrm{x}, \mathrm{y})$ is a quantity of local property of this point. When $\mathrm{T}$ depends only on the gray-level at that point, then it degenerates into a simple global threshold. Special attention needs to be given to the factor $\mathrm{p}(\mathrm{x}, \mathrm{y})$ - this is described as a property of the point. Actually, this is one of the more important components in the calculation of the threshold for a certain point. Let $f=\{f i j, i, j=1,2, \ldots . M\}$ denotes an $\mathrm{M} \times \mathrm{M}$ matrix of original image to be recovered and $\mathrm{M}$ is some integer power of 2. for the duration of the transmission, the signal $\mathrm{f}$ is corrupted by independent and identically distributed (i.i.d) zero mean, white Gaussian noise $n i j$ with standard deviation $\sigma$ i.e. $n i j ~ \sim$ $N(0, \sigma 2)$ and at the receiver end, the noisy observation $g i j=f i j+n i j$ is obtained. The ambition is to estimate the signal $\mathrm{f}$ from the noisy observations $g i j$ such that the Mean Square Error (MSE) is minimum. To achieve this, gij is transformed into wavelet domain, which decomposes gij into many subbands, which take apart the signal into so many frequency bands. The small coefficients in the subbands are dominated by noise, while coefficients with large unconditional value carry more signal information than noise. Replacing noisy coefficients by zero and an inverse wavelet transform may lead to reconstruction that has lesser noise. Normally Hard Thresholding and Soft Thresholding techniques are used for such denoising course of action. Hard and Soft thresholding with threshold $\lambda$ are defined as follows:

The hard thresholding operator is defined as $(U, \lambda)=U$ for all $|U|>\lambda=0$ otherwise

The soft thresholding operator on the other hand is defined as

$(U, \lambda)=\operatorname{sgn}(U) * \max (0,|U|-\lambda)$

We will then apply a set of filters on adaptive wavelet thresholding based denoised medical data in order to enhance results.

The set of filters we are going to use are

1.Median Filter

2.Average Filter

3.Diffusion Filter

We use these filters one by one after the adaptive threshold based wavelet decomposition.

\subsection{Median Filter}

Median filtering could be a nonlinear method constructive in reducing impulsive, or salt-and-pepper noise. it's additionally handy in protective edges in a picture whereas reducing random noise. Impulsive or salt-and pepper noise will occur as a result of a random bit error in an exceedingly channel. in an exceedingly median filter, a window slides on the image, and also the median passion price of the pels among the window becomes the output intensity of the pixel being processed. as an example, suppose the pel values among a window ar five, 6, 55, 10 and 15 , and also the pel being processed encompasses a price of fifty five. The output of the median filter and also the current pel location is ten, that is that the median of the 5 values.

\subsection{Average Filter}

The average, or box filter is the simplest of every one of filters. It substitute each pixel by the average of pixel values in a square centred at that pixel. All linear filters work in the equivalent way except that, instead of forming a simple average, a weighted average is formed. Using the vocabulary of Adaptive Wavelet Thresholding (Se 4.1.3), let $f i$, for $i, j=1,2, \ldots \ldots, n$ denote the pixel values in the image. We will use $g$, with pixel values $g i$, to denote the output from the filter. A linear filter of size $(2 m+1) \times(2 m+1)$, with specified weights $w k l$ for $k, l=-m, \ldots ., m$, gives.

gij $=\Sigma \Sigma w k l \quad f i+k,+l \quad m l=-m m k=-m \quad$ For $i, j=(m+1), \ldots \ldots,(n-m)$

For example, if $\mathrm{m}=1$, then the window over which averaging is carried out is $3 \times 3$, and $g i j=w-1,-1 \quad f i-1, j-1+w-1,0$ $f i-1, j+w-1,1 \quad f i-1, j+1+w 0,-1 \quad f i, j-1+w 0,0 \quad f i, j+w 0,1$ $f i, j+1+w 1,-1 f i+1, j-1+w 1,0 f i+1, j+w 1,1 f i+1, j+1$

For full generality, the weights (w) can depend on $i$ and $j$, resulting in a filter which varies across the image.

\subsection{Diffusion Filter}

A diffusion filter is a lucent photographic filter used for a special effect.Mathematically, this is described by Fick's law: $j$ $=-D \cdot \nabla u$

Where $\mathrm{j}$ is generated to recompense for the concentration gradient $\nabla u$. D is a tensor that describes the relation between them.

Now, using the Continuity Equation

(Conservation of mass): $\partial(u)=-\operatorname{div}(j)$

We get: $\partial(u)=\operatorname{div}(D . \nabla u)$

The explanation of the linear diffusion equation with a scalar diffusivity d

$\partial t(u)=\operatorname{div}(d \nabla u)$

Which is exactly the same operation as convolving the image $u$ with a Gaussian kernel of width $\sqrt{ } 2 t$.

\section{RESULT}

We implement our proposed method on different types of medical images like ultrasound,SPECT,MRI,CT,PET and calculate the efficiency terms of MSE and PSNR,SSIM,WPSNR.reultsed images are as follows-

\subsection{Results using MRI Image-}

\subsubsection{Salt \& Pepper Noise-}
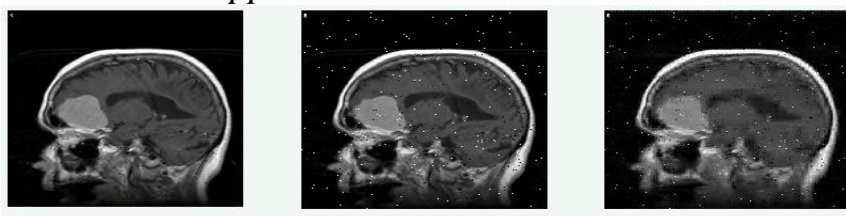

Origines

Hoised Medical Image

Denoised Medical Image

Denoised MRI image using Adaptive Wavelet Thresholding Algorithm
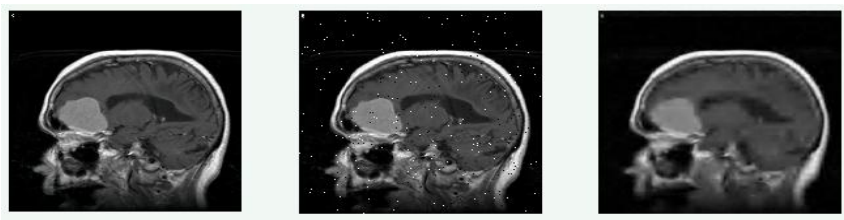

Original Medical Image

Hoised Medical Image

Denoised Medical Image

Hybrid Filters based denoised MRI image using Adaptive wavelet Thresholding algorithm

\subsubsection{Gaussion Noise-}

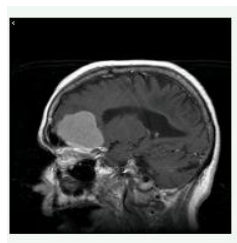

Original Medical Image

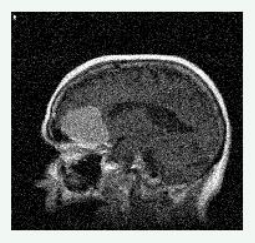

Hoised Medical Image

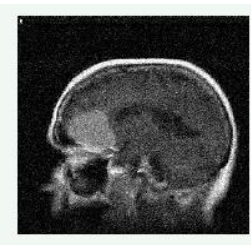

Denoised MRI image using Adaptive Wavelet Thresholding Algorithm 
Volume 83 - No3, December 2013

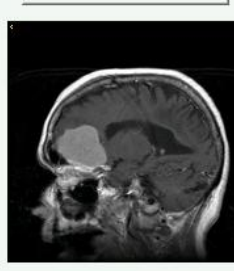

Original Medical Image

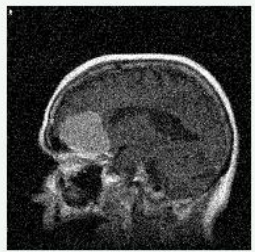

Hoised Medical Image

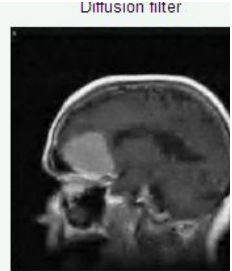

Denoised Medical Im

Hybrid Filters based denoised MRI image using Adaptive wavelet Thresholding algorithm

\subsubsection{Speckle noise-}

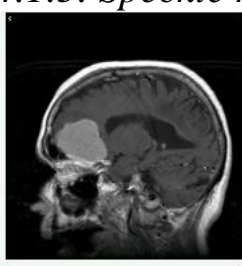

Original Medical Image

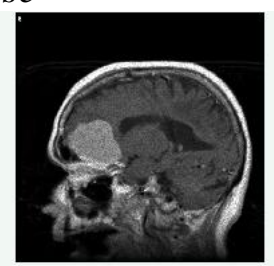

Hoised Medical Image

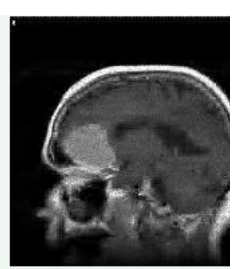

Denoised Medical Image

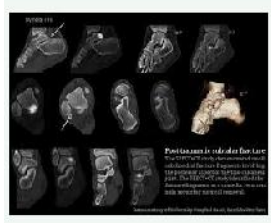

Original Medical Image

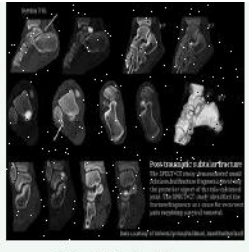

Hoised Medical Image

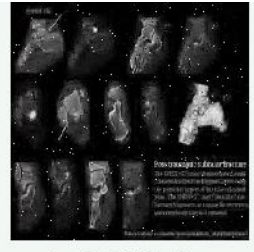

Denoised Medical Image

Denoised SPECT image using Adaptive Wavelet Thresholding Algorithm

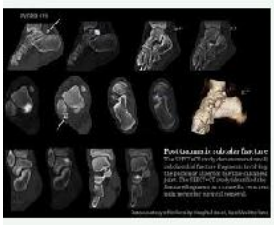

Original Medical Image

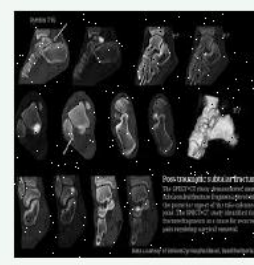

Hoised Medical Image

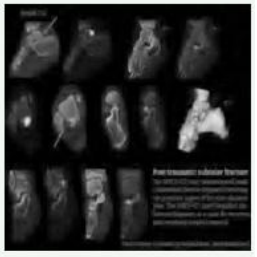

Denoised Medical Image

Hybrid Filters based denoised SPECT image using Adaptive wavelet Thresholding algorithm

Denoised MRI image using Adaptive Wavelet Thresholding Algorithm

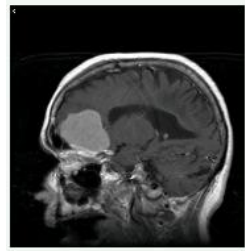

Original Medical Image

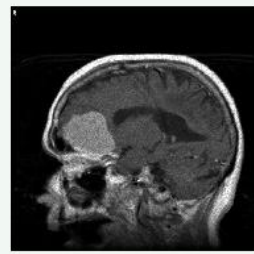

Hoised Medical Image

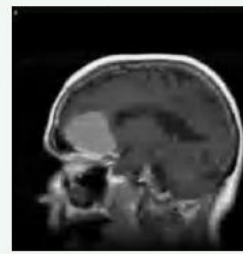

Denoised Medical Image

Hybrid Filters based denoised MRI image using Adaptive wavelet Thresholding algorithm

\subsubsection{Poission Noise-}

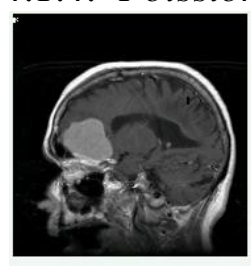

Original Medical Image

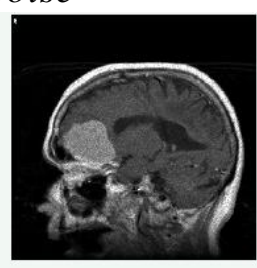

Hoised Medical Image

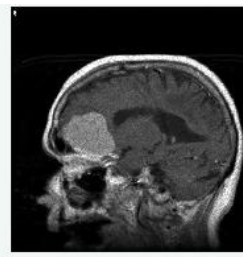

Denoised Medical Image

Denoised MRI image using Adaptive Wavelet Thresholding Algorithm

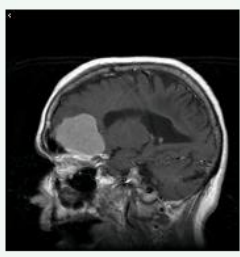

Original Medical Image

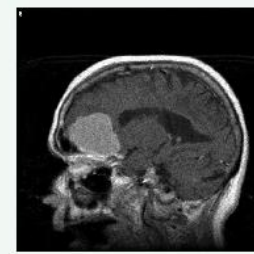

Noised Medical Image

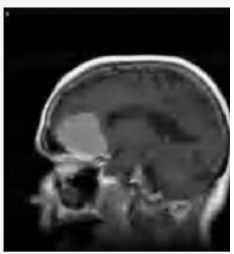

Denoised Medical Image

Hybrid Filters based denoised MRI image using Adaptive wavelet Thresholding algorithm

\subsection{SPECT Image-}

4.2.1.Salt \& Pepper Noise-

\subsubsection{Gaussion Noise-}
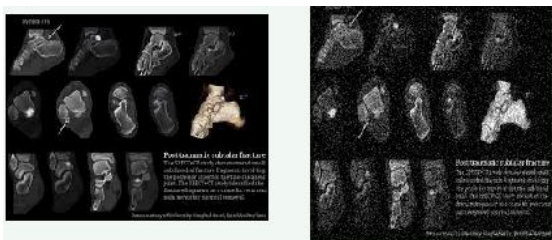

Hoised Medical Image

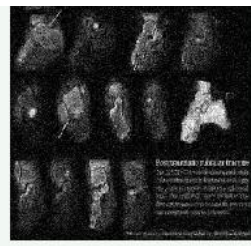

Denoised medical imege

Hybrid Filters based denoised SPECT image using Adaptive wavelet Thresholding algorithm

\subsubsection{Speckle Noise-}

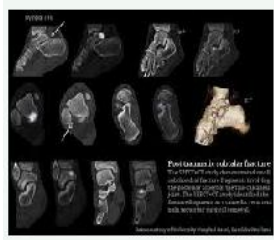

Original Medical Image

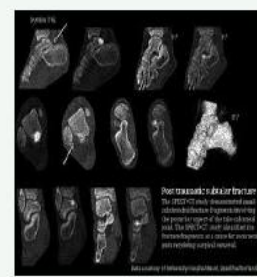

Hoised Medical Image

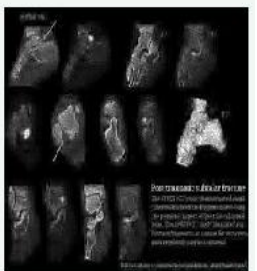

Denoised Medical Image

Denoised SPECT image using Adaptive Wavelet Thresholding Algorithm

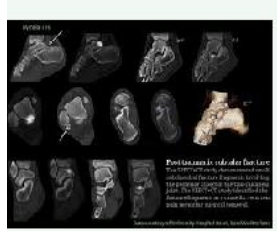

Original Medical Image

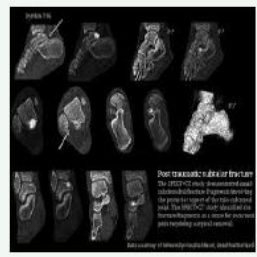

Hoised Medical Image

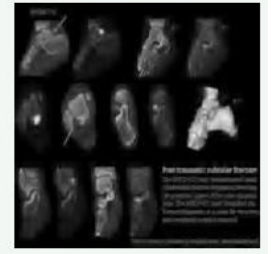

Denoised Medical Image

Hybrid Filters based denoised SPECT image using Adaptive wavelet Thresholding algorithm 


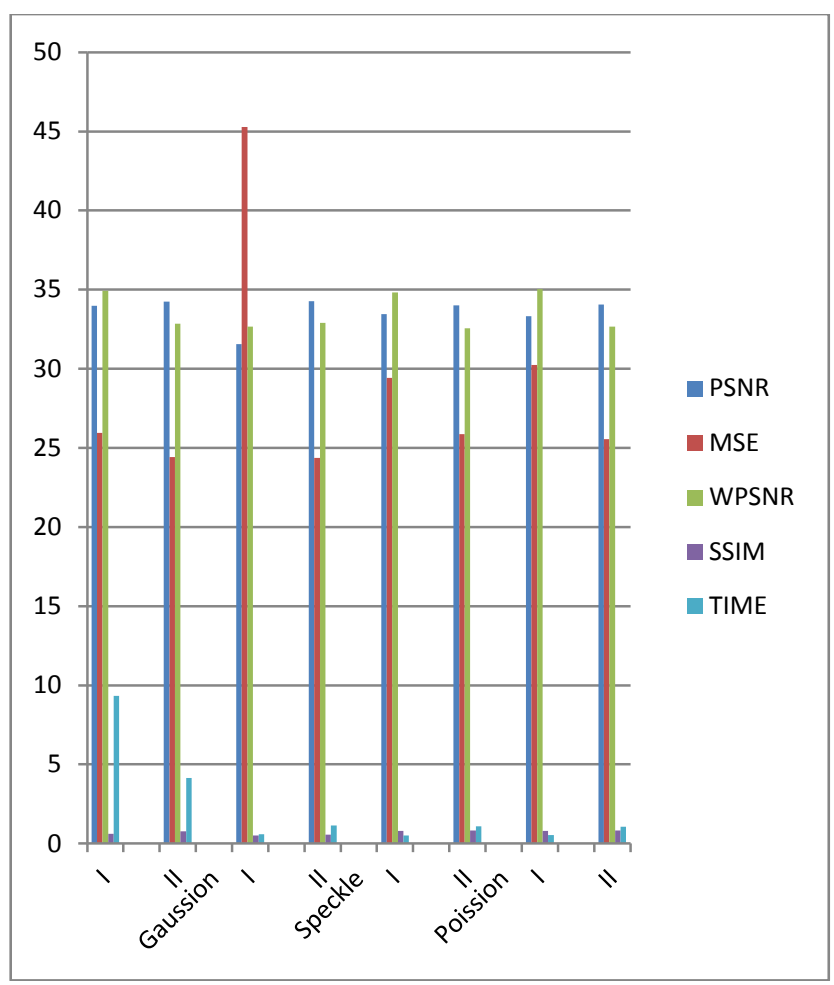

\subsection{Tables \& Graphs for Diffrent noise based results-}

\subsubsection{Table No 1 Results based on different parameters for MRI image}

\begin{tabular}{|l|l|l|l|l|l|}
\hline S\&p & PSNR & MSE & $\begin{array}{l}\text { WPSN } \\
\text { R }\end{array}$ & SSIM & TIME \\
\hline I & $\begin{array}{l}33.9 \\
8\end{array}$ & $\begin{array}{l}25.9 \\
6\end{array}$ & 34.93 & $\begin{array}{l}.617 \\
1\end{array}$ & 9.33 \\
\hline II & 34.2 & 24.4 & 32.85 & .779 & 4.15 \\
& 5 & 1 & & 0 & \\
\hline $\begin{array}{l}\text { Gaussio } \\
\text { n }\end{array}$ & & & & & \\
\hline I & 31.5 & 45.2 & 32.65 & $\begin{array}{l}.503 \\
8\end{array}$ & .577 \\
\hline II & 7 & 9 & & .568 & 1.147 \\
& 6 & 6 & 32.90 & 4 & \\
\hline Speckle & & & & & \\
\hline I & 33.4 & 29.4 & 34.83 & .809 & .5212 \\
& 4 & 3 & & 6 & \\
\hline II & 34.0 & 25.8 & 32.56 & .829 & 1.088 \\
& 0 & 6 & & 2 & 8 \\
\hline Poission & & & & & \\
\hline I & 33.3 & 30.2 & 35.03 & .809 & .5388 \\
& 2 & 3 & & 4 & \\
\hline II & 34.0 & 25.5 & 32.67 & .828 & 1.066 \\
& 5 & 6 & & 5 & 0 \\
\hline
\end{tabular}

Resulted Values \& Graph for MRI Images Using different Parameters

\subsubsection{Table No 2 Results based on different parameters for MRI image}

\begin{tabular}{|l|l|l|l|l|l|}
\hline S\&p & PSNR & MSE & WPSNR & SSIM & TIME \\
\hline I & 33.66 & 27.97 & 34.50 & .6161 & 1.657 \\
\hline II & 33.44 & 29.44 & 32.54 & .7582 & 1.757 \\
\hline Gaussion & & & & & \\
\hline I & 32.40 & 39.14 & 32.66 & .4980 & .6088 \\
\hline II & 33.44 & 29.44 & 32.54 & .7582 & 1.657 \\
\hline Speckle & & & & & \\
\hline I & 33.46 & 29.29 & 34.22 & .7974 & .5821 \\
\hline II & 33.24 & 30.82 & 32.21 & .7952 & 1.2665 \\
\hline Poission & & & & & \\
\hline I & 33.36 & 29.96 & 34.35 & .8030 & .5620 \\
\hline II & 33.27 & 30.61 & 32.31 & .7995 & 1.205 \\
\hline
\end{tabular}




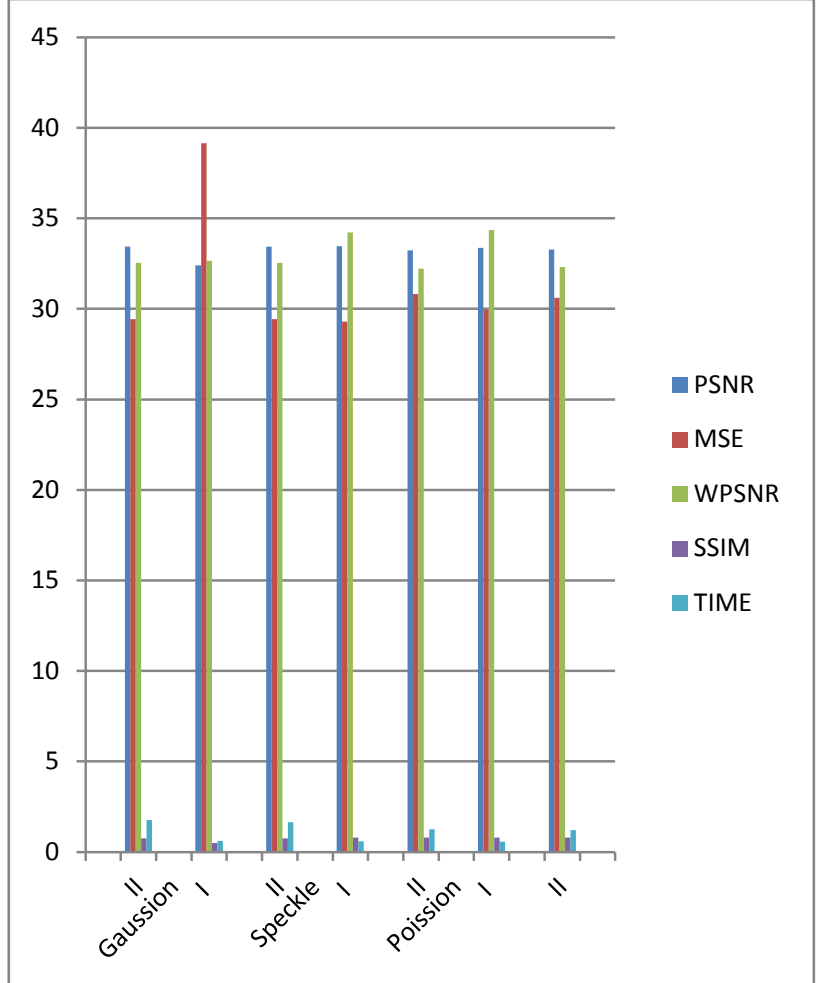

\section{Resulted Values \& Graph for SPECT Images Using different Parameters}

Problem with Mean-Squared Error (MSE) is that it depends strongly on the image intensity scaling. From table 2 it can be observed that image 2 under gaussion noise are more prone to MSE, as well speckle noise also reflects that image 2 are prone to MSE too.

PSNR avoids the problem of MSE by scaling the MSE according to the image range according to the image range which is represented by mathematical equation shown below.

$$
\mathrm{PSNR}=-10 \log _{10}\left(\mathrm{e}_{\mathrm{MSE}} / \mathrm{S}^{2}\right)
$$

Where $S$ is the maximum pixel value.

Greater PSNR means better restoration of the image, and is a good measure for comparing restoration results for the same image, but between images comparisions of PSNR are meaningless. So under the PSNR column for various image we can observe that image 1 is better restored in case of salt \& pepper noise, image 2 is better restored in case of gaussion noise, image 1 is better restored in case of speckle noise and in case of poission noise image 1 is better restored. Weighted peak signal to noise ratio (WPSNR) seems to have a very minor effect for any kind of noise under consideration as well as type of test images under observation, which we can clearly observe from table 1, 2and also from graphs 1,2.

Structural similarity index of image (SSIM) from above tables and graphs conclude that there is nearly any differences from the stuctural point of view, which mean that any of the noise present in the image harldy affects the structural composition of the images under test.

\section{CONCLUSION}

In image denoising, Adaptive Thresholding performed higher performance in each PSNR and visual quality than riffle denoising (hard thresholding or soft thresholding). The PSNR performance and visual quality will be increased by victimization quantity Shrinkage Functions primarily based Medical Image Denoising victimization distinct riffle rework.

\section{REFERENCES}

[1] Nilamani Bhoi, Dr. Sukadev Meher IEEE computer society, page no.20-25, ICETET 2008 IEEE, "Total Variation based Wavelet Domain Filter for Image Denoising"

[2] A. Beck, M. Teboulle, IEEE Transactions on Image Processing, vol. 18, no. 11, pp. 2419-2434, 2009. "Fast Gradient-Based Algorithms for Constrained Total Variation Image Denoising and Deblurring Problems,"

[3] T. Pock, A. Chambolle, D. Cremers, H. Bischof, IEEE Computer Society Conference on Computer Vision and Pattern Recognition, pp. 810-817, 2009. "A convex relaxation approach for computing minimal partitions,"

[4] Pierrick Coupé, Pierre Hellier, Charles Kervrann and Christian Barillot, IEEE transactions on image processing, vol. 18, no. 10, October 2009 "Nonlocal Means-Based Speckle Filtering for Ultrasound Images".

[5] Guodong Wang, Zhenkuan Pan, Zengfang Zhao, Xiaotong Sun, International Conference on Biomedical Engineering and Informatics (BMEI 2010) page 644-647,2010 IEEE "The Split Bregman Method of Image Decomposition Model for Ultrasound Image Denoising"

[6] S. Setzer, International Journal of Computer Vision, vol. 92, no 3, pp. 265-280, 2011, "Operator Splittings, Bregman Methods and Frame Shrinkage in Image Processing".

[7] Pascal Getreuer, Yale University Published in Image Processing On Line on may 2012. ISSN 2105-1232 (C)2012, "Rudin-Osher-Fatemi Total Variation Denoising using Split Bregman".

[8] Sachin Ruikar D D Doye 2010 International Conference on Mechanical and Electrical Technology (ICMET 2010), "Image Denoising Using Wavelet Transform".

[9] J S Bhat, B N Jagadale, Lakshminarayan $\mathrm{H}$ K, International Conference on Image and signal processing (ICSIP) 2010, "Image De-noising with an Optimal Threshold using Wavelets".

[10] LIU Wei, $2^{\text {nd }}$ International conference on image processing CISP 2009. "New Method for Image Denoising while Keeping Edge Information".

[11] S.Sudha , G.R.Suresh and R. Sukanesh, International Journal of Computer Theory and Engineering, Vol. 1, No. 1, pp.1793-8201, April 2009, "Speckle Noise Reduction in Ultrasound Images by Wavelet Thresholding based on Weighted Variance"

[12] Ioana Firoiu, Corina Nafornita, Jean-Marc Boucher, Alexandru Isar, IEEE transaction on instrument \& measurement, vol. 58, no. 8, august 2009, "Image Denoising Using a New Implementation of the Hyperanalytic Wavelet Transform".

[13] Li Hongqiao, Wang Shengqian, International Forum on Information Technology and Application 2009 "A New Image Denoising Method Using Wavelet Transform".

[14] S.Kother Mohideen, Dr. S. Arumuga Perumal, Dr. M.Mohamed Sathik, IJCSNS International Journal of Computer Science and Network Security, VOL.8 No.1, January 2008, "Image De-noising using Discrete Wavelet transform". 
[15] D. Giaouris, J.W. Finch School of Electrical, Electronic \& Computer Engineering, Newcastle University, Newcastle upon Tyne NE1 7RU, UK 2007 "Denoising using wavelets on electric drive applications".

[16] Eric J. Balster, Member, Yuan F. Zheng, Fellow, and Robert L. Ewing, Senior Member, IEEE Transactions On Image Processing, VOL. 14, NO. 12, DECEMBER 2005. "Feature-Based Wavelet Shrinkage Algorithm for Image Denoising".

[17] Eric J. Balster , Robert L. Ewing ,Yuan F. Zheng, IEEEtransaction on image processing, vol.14, no. 12, december 2005, "Feature-Based Wavelet Shrinkage Algorithm for Image Denoising" .

[18] Jean-Luc Starck, Jalal Fadili, and Fionn Murtagh IEEE TRANSACTIONS ON IMAGE PROCESSING, VOL. 16, "The Undecimated Wavelet Decomposition and its Reconstruction".

[19] D. Darian Muresan, Thomas W. Parks, "Adaptive Principal Components And Image Denoising".

\section{Authors Biblography-}

1.Mrs.Shruti Bhargava-

Mrs. Shruti Bhargava received her Bachelors degree in Electronics and Communication Engineering in the year 2007 and Masters degree in Digital Communication in the year 2010 from R.G.P.V ,Bhopal(M.P)India. Currently she is pursing her $\mathrm{PhD}$ from the D.K. N.M.U. Her research interests are Image processing and Digital Communication.

\section{Dr. Ajay Somkuwar-}

Dr. Ajay Somkuwar received BE with honors from Jabalpur Engineering College and $M$. Tech. degree in Digital Communication Engineering from MACT Bhopal, subsequently he carried out his research form Indian Institute of Technology, New Delhi and awarded Ph.D. in 2003. Presently he is working as Professor in the Department of Electronics and Communication at Maulana Azad National Institute of Technology, Bhopal. He has published more than 100 papers of national and International repute. $\mathrm{He}$ has been Member/ Chairman of many selection committees for recruitment of staff and faculty. His research areas include signal processing Image processing and Biomedical Engineering. He has produced 5 Ph.D. degrees. He is member of IETE, New Delhi and International Association of Engineers (IAENG). Recently he is awarded by "Indira Gandhi Shiromani award-2011" for his contribution to nation. He also awarded by Best Citizen of India-2012. and "Siksha Rattan Award- 2012" 